\title{
Medical Image of the Week: Nocardiosis
}

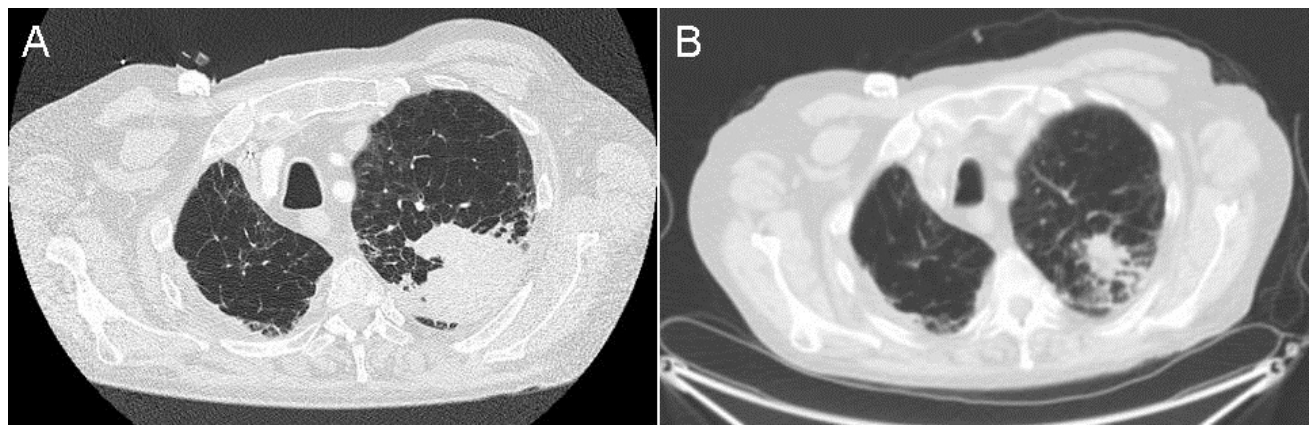

Figure 1. Panel A: Thoracic CT scan showing enlarged left upper lobe mass. Panel B: CT scan from one month earlier showing a smaller lesion.

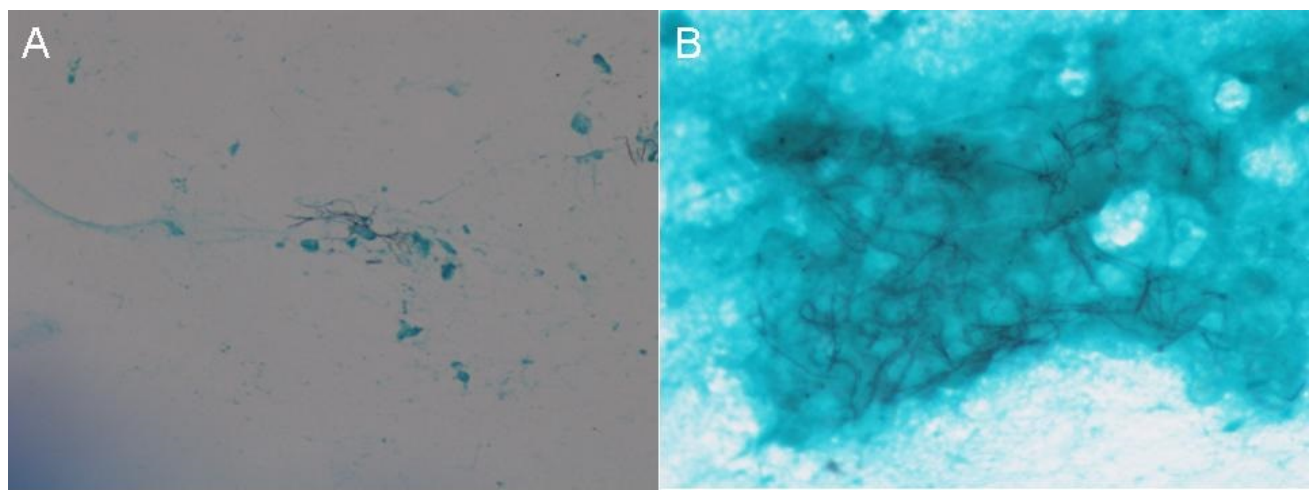

Figure 2. Panel A: GMS Silver stain showing Nocardia (200X magnification). Panel B: GMS silver stain showing Nocardia (400X magnification).

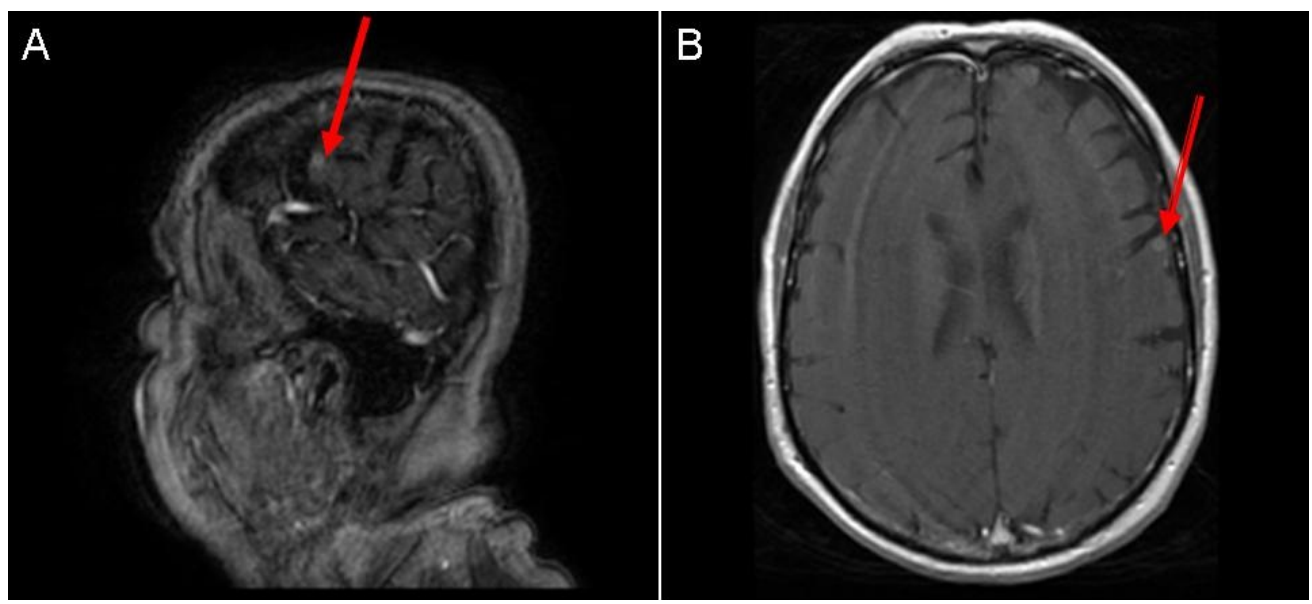

Figure 3. MRI Brain with arrows pointing to the lesion. 
A 67 year-old man with advanced adenocarcinoma of the lung on chemotherapy and severe steroid dependent chronic obstructive pulmonary disease (COPD) was admitted for treatment of acute on chronic respiratory failure. He was admitted to the intensive care unit and required non-invasive positive pressure ventilation. He had a chest computed tomography scan (Figure 1A), with a left upper lobe mass, which was significantly larger than noted on a previous PET/CT scan (Figure 1B) from one month ago. He was placed on empiric broad-spectrum antibiotics and clinically improved. He underwent a transthoracic lung biopsy (Figure 2), which revealed the presence of organisms consistent with Nocardia on silver stain. A brain MRI (Figure 3) showed the presence of a 4 $\mathrm{mm}$ enhancing lesion likely consistent with Nocardia.

Nocardiosis is a gram-positive bacterial infection caused by aerobic actinomycetes and is an important opportunistic pulmonary infection. It should be considered in the differential diagnosis of pulmonary infiltrates in immunosuppressed patients, including those with neoplasms, after organ transplantation, advanced HIV disease and those receiving chronic corticosteroid therapy or chemotherapy (1). Of importance to pulmonologists, in two reviews, COPD was a common underlying condition, representing over $20 \%$ of patients with Nocardiosis in these reports $(2,3)$. Nocardia species are found in soil and infection is generally acquired through inhalation. The most common symptoms are fever, cough, pleuritic chest pain and headache. Common chest radiographic findings include consolidation, nodules, cavities and pleural effusions. Nocardia infections can disseminate to any organ but it has a predilection for spread to the central nervous system and patients with pulmonary Nocardia infections should have brain imaging to evaluate for cerebral dissemination. Antibiotics that are typically effective in Nocardia infections include trimethoprim-sulfamethoxazole (TMP-SMX), imipenim, amikacin, ceftriaxone and cefotaxime. However, antibiotic susceptibilities should be obtained and treatment tailored accordingly. It is recommended to treat severe systemic infections with two or three intravenous agents while awaiting susceptibility results. Treatment is usually prolonged because of the tendency of Nocardia infections to relapse or progress. For patients with serious pulmonary infections and immunocompromised patients, duration of therapy is often at least 6 to 12 months or longer. Our patient was treated with TMPSMX and meropenem and clinically improved. His steroids were rapidly tapered. Sputum cultures grew Nocardia farcinica.

Aarthi Ganesh MD, Muna Omar MD, James Knepler MD, and Linda Snyder MD Department of Pulmonary and Critical Care Banner University Medical Center

Tucson, AZ

\section{References}

1. Grigor LM, Hoover SE. Nocardiosis at a university medical center in the American southwest. Infect Dis Clin Pract 2014:22:279-82. [CrossRef] 
2. Minero MV, Marín M, Cercenado E, Rabadán PM, Bouza E, Mu-oz P. Nocardiosis at the turn of the century. Medicine (Baltimore). 2009;88(4):250-61. [CrossRef] [PubMed]

3. Martínez Tomás R, Menéndez Villanueva R, Reyes Calzada S, Santos Durantez M, Vallés Tarazona JM, Modesto Alapont M, Gobernado Serrano M. Pulmonary nocardio-sis: risk factors and outcomes. Respirology. 2007;12(3):394-400. [CrossRef] [PubMed] 